\title{
Immunohistochemical CD10 Expression is Useful for Differentiating Malignant Melanoma from Benign Melanocytic Nevus
}

\author{
Masahiro Hoshikawa $^{1}$, Hirotaka Koizumi ${ }^{1}$, Rumiko Handa ${ }^{1}$, \\ Saeko Naruki ${ }^{1}$, Junki Koike ${ }^{2}$, and Masayuki Takagi ${ }^{1}$
}

(Received for Publication: April 27, 2015)

\begin{abstract}
Malignant melanoma (MM), an aggressive skin neoplasm, can be difficult to differentiate histologically from benign melanocytic nevi (BMN). Although immunohistochemical analysis could facilitate diagnosis of $\mathrm{MM}$, markers that discriminate between benign and malignant lesions have not yet been established. However, CD10 expression is associated with MM progression. We immunostained $36 \mathrm{MM}$ and $50 \mathrm{BMN}$ specimens for CD10 to evaluate whether CD10 immunostaining could distinguish between BMN and MM tumors. In the 36 MM samples, we found CD10 expression in 17 (47.2\%) sample tumor cells and 32 (88.9\%) stromal cells, for 34 of the $36 \mathrm{MM}$ specimens (94.4\%) overall. In contrast, no BMN (0/50) samples had CD10+ tumor cells, although a few $(8 / 50,16.0 \%)$ showed stromal staining. The two specimen types thus significantly differed in CD10 expression $(P<0.01)$, indicating that melanocytic $\mathrm{CD} 10^{+}$tumor cells are likely to be malignant. Also, melanocytic stromal cells with diffuse, strong CD10 expression are more likely to be malignant than those with light, focal CD10 expression. Although hematoxylin and eosin staining is fundamental to the diagnosis of melanocytic lesions, CD10 immunostaining may help distinguish between BMN and MM.
\end{abstract}

\section{Key words}

CD10, immunohistochemistry, malignant melanoma, melanocytic nevus

\section{Introduction}

Malignant melanoma (MM), an aggressive skin neoplasm with poor prognosis, is diagnosed by clinical and pathological findings. In the clinical arm, use of dermatoscopy to diagnose MM has improved in recent years ${ }^{122}$. Similarly, immunohistochemistry (IHC) and molecular analyses are the leading developments in pathological diagnosis. However, histopathological diagnoses of melanocytic lesions are occasionally challenging. Although clearly aggressive MMs can be diagnosed without difficulty, MMs with equivocal invasiveness, such as in situ lesions, or those with similar morphology to melanocytic nevi (BMN) are often problematic. IHC is a useful tool, but diagnostic panels for differentiating MM from
BMN are controversial ${ }^{3-5)}$. Representative melanocytic markers, including S100, HMB- 45, and melan$\mathrm{A}$, are often used in diagnosing melanocytic lesions; although they are useful for MM and tumors with melanocytic differentiation ${ }^{3)}$, their use in distinguishing malignant and benign melanocytic lesions is limited $^{3)}$.

CD10 expression can reportedly predict aggressiveness of $\mathrm{MM}^{6-9)}$. CD10 is a cell surface glycoprotein expressed in both normal and neoplastic cells, including follicular lymphoma ${ }^{10)}$, renal cell carcinoma ${ }^{11)}$, endometrial stromal sarcoma ${ }^{11) 12}$, atypical fibroxanthoma ${ }^{13)}$, and many benign or malignant tumors ${ }^{11) 13}$, although the precise function of CD 10 in each tumor is still not fully understood ${ }^{14}$.

CD10 expression in MM was first described by

1 Department of Pathology, School of Medicine, St. Marianna University

2 Department of Pathology, Kawasaki Municipal Tama Hospital 
Jongeneel et al. ${ }^{15)}$ using flow cytometry in cell lines. Carrel et al. ${ }^{16) 17}$ ) then showed CD10 expression in vivo in MM tissues by IHC; several other reports have significantly correlated CD10 expression levels with tumor progression ${ }^{68)(18-20)}$.

Although a number of studies have shown CD10 expression in MM, very little is known about its expression in melanocytic nevus, a benign counterpart of $\mathrm{MM}^{20)}$. In this study, we compared IHC expression patterns of CD10 in MM and melanocytic nevus and examined the usefulness of CD10 in differential diagnosis of these lesions.

\section{Materials and methods}

We retrieved specimens from the pathology database in St. Marianna University Hospital, all of which were fixed with formalin and embedded in paraffin. We selected typical BMN specimens with wide dermal spreading (i.e. intradermal or compound type, $n=50$ ) and cutaneous MM with unequivocal dermal invasion $(n=36)$. Morphological diagnoses of these lesions were confirmed by at least two pathologists.

We performed IHC staining on 3- $\mu$ m-thick paraffin sections. Melanin bleaching with warm diluted hydrogen peroxidase was performed on some samples with massive pigmentation ${ }^{21}$. Sections were incubated for 2 hours at room temperature with a monoclonal mouse anti-human CD10 antibody (clone: 56C6, 1:2 dilution, Nichirei, Tokyo). Reacted antibodies were detected by Simple Stain MAX-PO (Nichirei, Tokyo, Japan). We scored CD10 expression as $3+: \geq 50 \%$ of tumor cells CD10+; $2+: 10-50 \%$ of tumor cells $\mathrm{CD}^{+}$; and $1+:<10 \%$ of tumor cells $\mathrm{CD} 10^{+}$. In stromal cells, CD10 expression was evaluated as diffuse, focal, or negative. We analyzed correlation of CD10 expression between MM and BMN using Fischer's exact test. $P<0.05$ was considered to be significant. We also analyzed sensitivity, specificity, positive predictive value (PPV), and negative predictive value (NPV) of CD10 expression in MM and BMN, as described elsewhere ${ }^{6222}$.

This study was approved by the Institutional Ethics Committee of St. Marianna University School of Medicine (approval no. 2797).

\section{Results}

The 36 patients with MM ranged in age from 4 to 89 years old $(63.8 \pm 17.7$, mean \pm S.D). Their MM subtypes were 12 cases (33.3\%) of acral lentiginous melanoma; $19(52.8 \%)$ of nodular melanoma; and 5 $(13.9 \%)$ of superficial spreading melanoma (Table
1). The 50 patients with $\mathrm{BMN}$ ranged in age from 7 to 75 years $(41.1 \pm 16.6)$.

Morphologically, representative MM cells had pleomorphic and eosinophilic cytoplasm and ovalshaped nuclei with prominent nucleoli (Fig. 1a), whereas BMN were composed of relatively monotonous small round cells with fine nuclei. No maturation was seen in MM, but typical BMN cells showed maturation according to the depth of dermis.

Among the tumor cells in the $36 \mathrm{MM}$ samples, 17 were $\mathrm{CD}_{10} 0^{+}(47.2 \%$; Fig. 1 b-d), whereas all 50 BMN samples were CD10- $(P<0.01$; Table 2; Figures 1 and 2). Of these $17 \mathrm{MM}$ samples with $\mathrm{CD} 10^{+}$ tumor cells, 6 scored $3+(16.7 \%$, Fig. 1b); 5 scored $2+(13.9 \%$, Fig. 1c); and 6 scored $1+(16.7 \%$, Fig. 1d). We also found CD10 expression in stromal cells in 32 of $36 \mathrm{MMs}(88.9 \%$, Fig. 1f, h), with intratumoral spreading in 4 cases $(11.1 \%$; intratumoral pattern, Fig. 1e, f), peritumoral proliferation in 16 cases (44.4\% peritumoral pattern, Fig. 1g, h); and combined intra- and peritumoral patterns in 12 cases $(33.3 \%)$. In contrast, stromal cells in only 8 of 50 BMN samples expressed CD10 (16.0\%), all in intratumoral patterns (Figure 2); six had $\mathrm{CD} 10^{+}$stromal cells located near deep dermal vessels (Fig. 2a, b), one had $\mathrm{CD} 10^{+}$stromal cells widely spread from the hair follicles and vessels (Fig. 2c, d), and the remaining case had relatively diffuse $\mathrm{CD} 10^{+}$stromal expression through the lesion. This last case was associated with a skin ulcer and infiltration of $\mathrm{CD}^{+} 0^{+}$granulocytes (Fig. 2e, f). In BMN, CD10+ stromal cells were focal and thin or fine in nevi compared with a diffuse, thick pattern in MMs.

The $\mathrm{CD} 10^{+}$tumor and stromal cells combined marked $34 \mathrm{MM}$ cases (94.4\%), compared with 8 BMN cases $(16.0 \% ; P<0.01)$. In MM tumor cells, this method was $47 \%$ sensitive, $100 \%$ specific with $100 \%$ PPV and 73\% NPV (Table 3), but 0\% sensitive, 53\% specific, with 0\% PPV and 28\% NPV for BMN tumor cells. For $\mathrm{CD} 10^{+}$tumor and stromal cells combined, results were $94 \%$ sensitive and $84 \%$ specific with $81 \%$ PPV and 96\% NPV for MM, and 16\% sensitive and 6\% specific with 19\% PPV and 5\% NPV for BMN.

\section{Discussion}

Invasive $\mathrm{MM}$ is an aggressive skin neoplasm that shows downward dermal infiltration with destruction of skin appendages, vessels, and interstitial stromal fibroblasts, whereas BMN may spread in the dermis without destruction of dermal structures. Al- 
Table 1. Clinicopathological Features of Patients with Nevi or Melanoma

\begin{tabular}{llcc}
\hline & & Melanoma & Nevus \\
\hline Sex & Male & $19(52.8 \%)$ & $19(38.0 \%)$ \\
& Female & $17(47.2 \%)$ & $38(76.0 \%)$ \\
\hline Age (years) & Range & $4-89$ & $7-75$ \\
& Mean \pm SD & $63.8 \pm 17.7 *$ & $41.1 \pm 16.6^{*}$ \\
\hline Site & Head and neck & $4(11.1 \%)$ & $38(76.0 \%)$ \\
& Trunk & $3(8.3 \%)$ & $11(22.0 \%)$ \\
& Genital area & $4(11.1 \%)$ & $0(0 \%)$ \\
Subtype & Extremity & $25(69.4 \%)$ & $1(2 \%)$ \\
\hline ALM & $12(33.3 \%)$ & $\mathrm{n} / \mathrm{a}$ \\
& NM & $19(52.8 \%)$ & $\mathrm{n} / \mathrm{a}$ \\
& SSM & $5(13.9 \%)$ & $\mathrm{n} / \mathrm{a}$ \\
\hline Thickness (mm)* level & II & $1(2.8 \%)$ & $\mathrm{n} / \mathrm{a}$ \\
& Range & $1.0-13.0$ & $1.2-6.7$ \\
& Mean \pm SD & $4.3 \pm 2.7$ & $3.0 \pm 1.1$ \\
\hline & IV & $20(55.6 \%)$ & $\mathrm{n} / \mathrm{a}$ \\
& V & $6(16.7 \%)$ & $\mathrm{n} / \mathrm{a}$ \\
\hline
\end{tabular}

ALM: acral lentigenous melanoma; MM: malignant melanoma; n/a: not applicable; NM: nodular melanoma; SSM: superficial spreading melanoma.

*Thickness refers to Breslow's scale and is also applied to nevi.

Table 2. Results of Immunohistochemistry for CD10 in Tumor and/or Stromal Cells

\begin{tabular}{|c|c|c|c|c|}
\hline & MM & Nevus & Total & $\mathrm{p}$-value \\
\hline \multicolumn{5}{|l|}{ Tumor cells } \\
\hline $\mathrm{CD} 10+$ total & $17(47.2 \%)$ & $0(0 \%)$ & 17 & \\
\hline $3+$ & $6(16.7 \%)$ & $0(0 \%)$ & & \\
\hline $2+$ & $5(13.9 \%)$ & $0(0 \%)$ & & \\
\hline $1+$ & $6(16.7 \%)$ & $0(0 \%)$ & & \\
\hline CD10- & $19(52.7 \%)$ & $50(100 \%)$ & 69 & $<0.01 *$ \\
\hline \multicolumn{5}{|l|}{ Stromal cells } \\
\hline $\mathrm{CD} 10+$ total & $32(88.9 \%)$ & $8(16.0 \%)$ & 40 & \\
\hline intratumoral pattern & $4(11.1 \%)$ & $8(16.0 \%)$ & & \\
\hline peritumoral pattern & $16(44.4 \%)$ & $0(0 \%)$ & & \\
\hline both patterns & $12(33.3 \%)$ & $0(0 \%)$ & & \\
\hline CD10- & $4(11.1 \%)$ & $42(84.0 \%)$ & 46 & $<0.01^{*}$ \\
\hline \multicolumn{5}{|l|}{ Either tumor or stromal cells } \\
\hline $\mathrm{CD} 10+$ & $34(94.4 \%)$ & $8(16.0 \%)$ & 42 & \\
\hline CD10- & $2(0.05 \%)$ & $42(84.0 \%)$ & 44 & $<0.01^{*}$ \\
\hline
\end{tabular}

*Fischer's exact test 

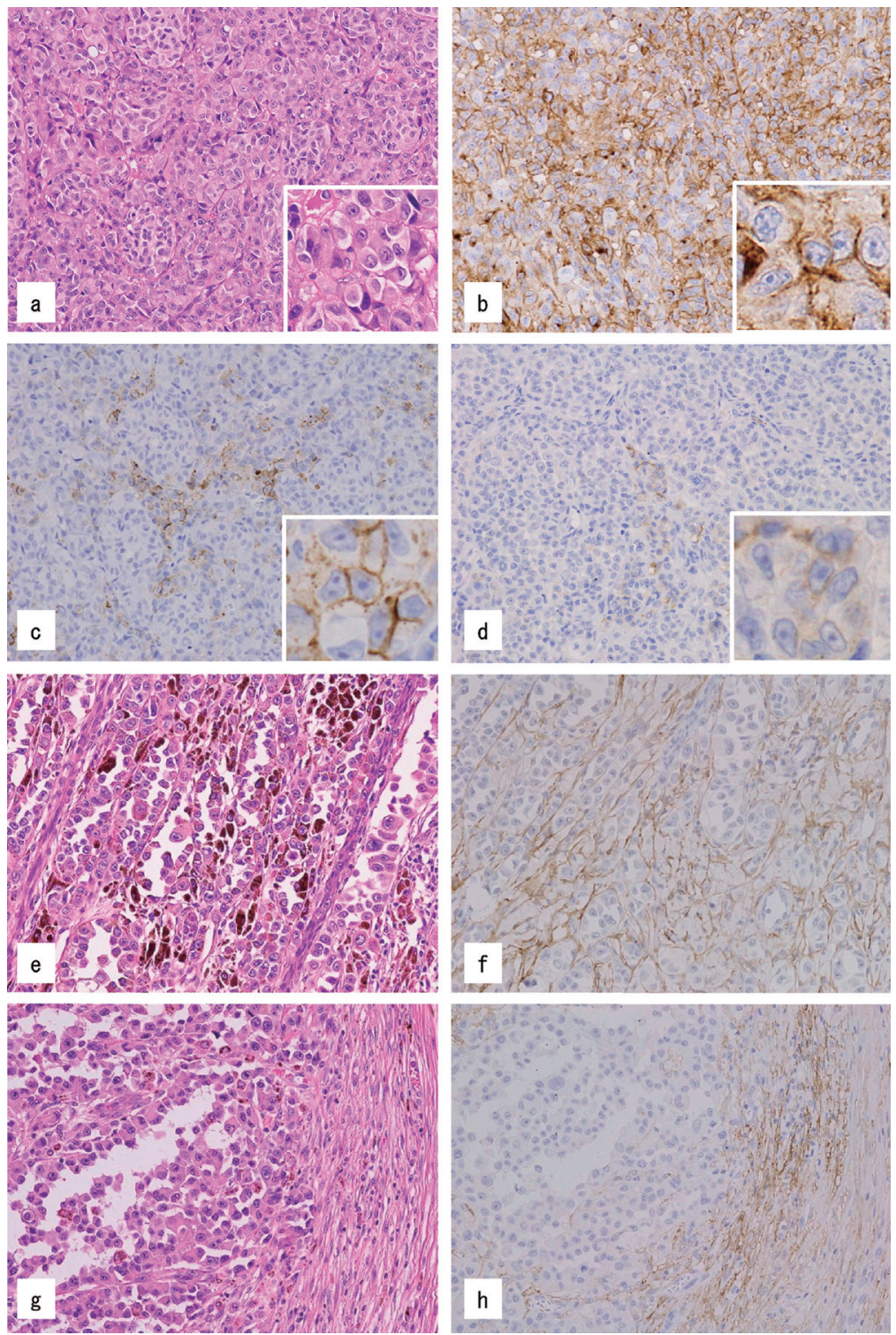

Figure 1. Immunohistochemical CD10 expression in malignant melanoma (MM)

a. Representative pleomorphic MM. b. Diffuse 3+ CD10 expression in MM cells. c. CD10 expression, 2+, seen in less than $50 \%$ of MM cells. d. A few $\mathrm{CD} 10^{+}$tumor cells, 1+. e. MM with intratumoral or intercellular stromal cells. f. Intratumoral proliferation of CD10 ${ }^{+}$stromal cells. g. MM with peritumoral proliferation of fibrous stromal tissue (fibroblast). h. Peritumoral expression of $\mathrm{CD} 10^{+}$stromal cells. Magnification: $200 \times$ for $\mathbf{a - h}$; $400 \times$ for inset of a-d.

though benign and malignant melanocytic lesions essentially differ, those with wide dermal involvement can be difficult to distinguish morphologically. Use of IHC to diagnose melanocytic lesions ${ }^{3-5)}$ also did not completely present a clear way to discriminate between these lesions.

CD10, also known as neutral endopeptidase, enkephalinase, neprilsin, or common acute lymphoblastic leukemia antigen (CALLA), is a zinc-dependent metalloproteinase located on the cell surface ${ }^{14)}$ and is 

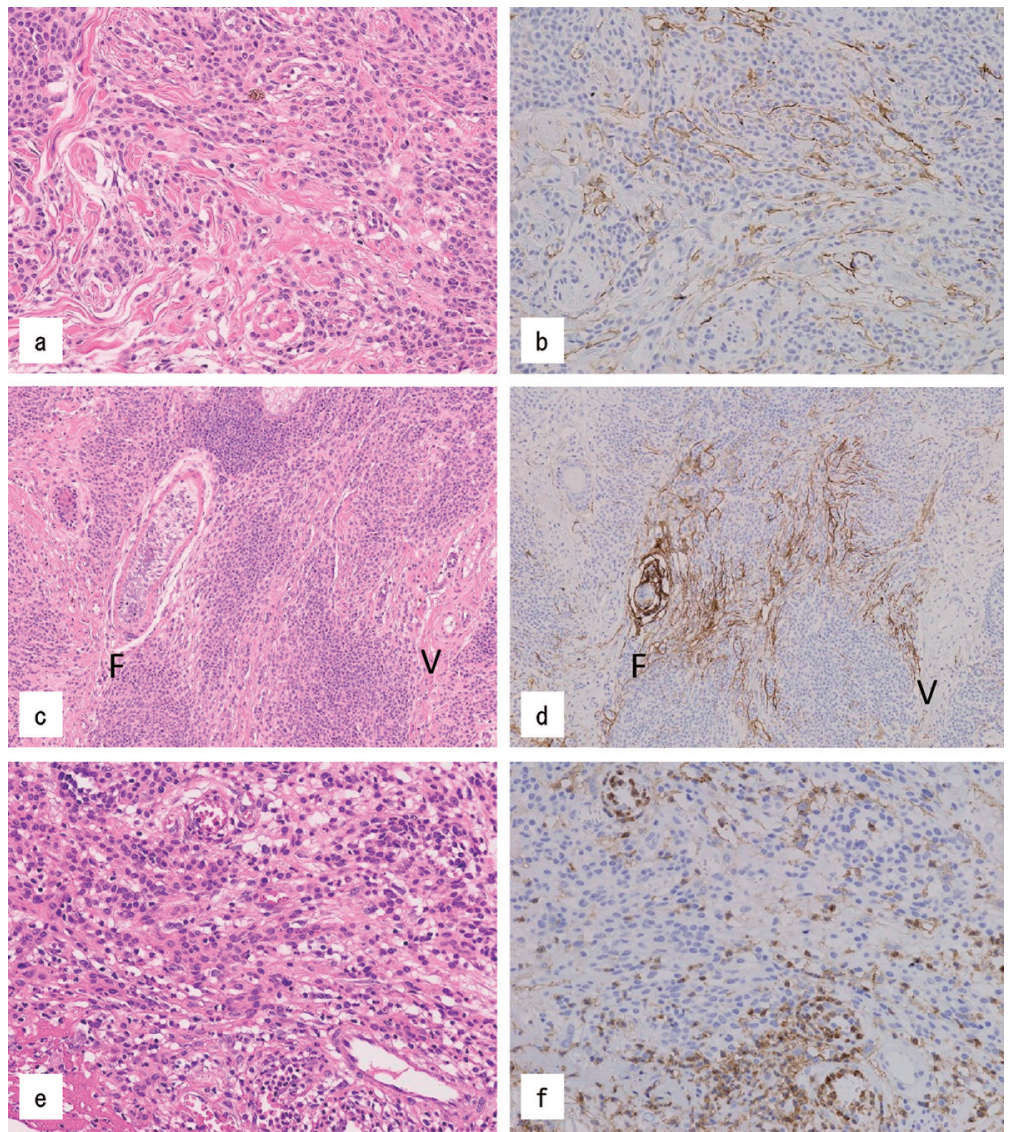

Figure 2. Nevus with $\mathrm{CD} 10^{+}$stromal cells. a. Pigmented nevus in deep dermis. b. $\mathrm{CD}_{10}{ }^{+}$fine stromal cells near vessels in the deep dermis. c. Nevus cells around hair follicles $(\mathrm{F})$ and vessels $(\mathrm{V})$. d. $\mathrm{CD} 10^{+}$stromal cells extended from around the hair follicles and vessels. e. Nevus with granulocytic infiltration. f. Fine $\mathrm{CD} 10^{+}$stromal cells between nevus cells, associated with infiltrating $\mathrm{CD}^{+} 0^{+}$granulocytes. Magnification: $200 \times$ for $\mathbf{a}$, b, e, f; $100 \times$ for $\mathbf{c}, \mathbf{d}$.

Table 3. Sensitivity, Specificity, PPV, and NPV for CD10 Expression in Melanocytic Lesions

\begin{tabular}{lcccc}
\hline & \multicolumn{2}{c}{ Tumoral cells (\%) } & \multicolumn{2}{c}{ Tumoral and/or stromal cells (\%) } \\
\cline { 2 - 5 } & MM & Nevus & MM & Nevus \\
\hline Sensitivity & 47 & 0 & 94 & 16 \\
Specificity & 100 & 53 & 84 & 6 \\
PPV & 100 & 0 & 81 & 19 \\
NPV & 73 & 28 & 96 & 5 \\
\hline
\end{tabular}

PPV: positive predictive value; NPV: negative predictive value. 
expressed in many normal cells, including myoepithelial breast cells ${ }^{23)}$, proximal uriniferous tubules ${ }^{24)}$, stromal endometrial cells ${ }^{25}$, and stromal cells of skin appendages ${ }^{18)}$, as well as many benign or malignant tumors ${ }^{1113)}$. Chu et al. ${ }^{11)}$ found CD10 expressed in renal cell carcinoma, transitional cell carcinoma, prostatic adenocarcinoma, endometrial stromal sarcoma, rhabdomyosarcoma, and schwannoma. Hematopathological malignancies, including acute lymphoblastic leukemia, follicular lymphoma, Burkitt lymphoma, and angioimmunoblastic T-cell lymphoma, also express CD10 10226), with diffuse cytoplasmic and/or membranous IHC staining patterns. For MM, Carrel et al. ${ }^{16) 17)}$ showed CD10 expression in $63 \%$ and $38 \%$ of ocular and cutaneous tumor tissues, respectively. Kianitakis et al. ${ }^{6}$ found that frequency of CD10 expression in MMs was higher in metastatic lesions in skin $(69.0 \%)$ or lymph nodes $(61.0 \%)$ than in primary tumors $(21.4 \%)$, in accordance with several other reports $^{7-9)}$. These results suggest a close relationship between up-regulation of CD10 and tumor progression in MM. However, CD10 expression in BMN, a benign counterpart to MM, has not been fully explained $^{20)}$. To evaluate the usefulness of CD10 IHC to distinguish malignant and benign melanocytic lesions, we studied CD10 expression patterns in primary cutaneous MMs and BMN.

In primary cutaneous MMs, tumor cell expression ranged from $3.0-53.8 \%$ in previous reports ${ }^{7-9) 20127)}$. In this study, CD10+ tumor cells were seen in 17 of 36 cases (47.2\%), for which this assay was $47 \%$ sensitive and $100 \%$ specific, with $100 \%$ PPV and $73 \%$ NPV. As this method was not highly sensitive for the diagnosis of MMs, CD10 should not be a representative tumor marker in MM. However, as no BMN cells produced CD10 antigen, this test was $100 \%$ specific and had a $100 \%$ PPV in this study, which suggests that CD10 could be a useful marker for malignancy.

CD10 expression has not been widely studied in BMN. Oba et al. ${ }^{20)}$ reported a CD10 expression study relating to tumor progression and prognosis of MMs compared with melanocytic nevi. They reported 34 of $64(53 \%)$ primary MM specimens and 4 of $40(10 \%)$ BMN specimens expressed CD10, similar to our results in the present study, although four melanocytic nevi (2 Spitz and 2 compound nevi) were $\mathrm{CD} 10^{+}$. The $\mathrm{CD} 10^{-}$specimens in this investigation were 14 compound, 14 junctional, 4 Spitz, and 4 dysplastic nevi; Spitz nevi were suggested to be more likely to express CD10 compared with common or dysplastic nevi. Spitz and dysplastic nevi are special BMN types that could be difficult to distinguish from melanoma, and warrant further study. Although Oba et al. ${ }^{20)}$ found common BMN could express CD10, we found no $\mathrm{CD} 10^{+} \mathrm{BMN}$ tumoral cells in the present study. Additional BMN specimens are needed to clarify the expression of CD10 in common melanocytic nevi, as well as Spitz and dysplastic nevi.

Stromal proliferation is not unusual in invasive malignant neoplasms. Proliferation of $\mathrm{CD} 10^{+}$stromal cells has been reported in other organs. Stromal expression of CD10 in invasive breast carcinoma is reportedly associated with increasing tumor grade and worsening prognosis ${ }^{28-30}$. Ogawa et al. ${ }^{31)}$ showed that $\mathrm{CD} 10^{+}$stromal cells in colorectal carcinoma were associated with tumor progression. As $\mathrm{CD} 10^{+}$stromal cells are frequently seen near or at the invasive fronts of tumors, CD10 may be involved in the interaction between neoplastic cells and stromal cells ${ }^{28-31}$. In this study, two types of $\mathrm{CD} 10^{+}$stromal cell reactions were found in MMs, namely intratumoral proliferation pattern (Fig. 1e, f) and peritumoral proliferation pattern (Fig. 1g, h). Bialovic et al. ${ }^{7}$ first described these two types of stromal cells and associated $\mathrm{CD} 10^{+}$stromal cells with tumor progression. In MMs, neoplastic cells and $\mathrm{CD} 10^{+}$stromal cells appear to have some relationship. Among the 50 nevus samples, 8 (16.0\%) had $\mathrm{CD} 10^{+}$stromal cells, of which 7 showed only a focal and fine positivity with the positive cells located in the perivascular areas of deep dermis (Fig. 2a-d). The eighth specimen showed relatively diffuse positivity in the lesion and was associated with a dermal ulcer with diffuse infiltration of inflammatory cells. In some of our MM specimens, $\mathrm{CD} 10^{+}$stromal cells tended to proliferate around vessels near skin ulcers, suggesting that $\mathrm{CD}^{+} 0^{+}$stromal cells around vessels and skin appendages may increase under the influence of inflammation. The function of $\mathrm{CD} 10^{+}$stromal cells in tumors has not been widely studied. Xie et al. ${ }^{32)}$ reported that the $\mathrm{CD} 10^{+}$fibroblasts inhibited the invasive capacity of interleukin- $1 \alpha$-producing squamous cell carcinoma by diminishing substance P. As infiltration of peritumoral $\mathrm{CD} 10^{+}$stromal cells in squamous cell carcinoma is similar to that seen in $\mathrm{CD} 10^{+}$ stromal cells in MM, they could share similar functions as inhibitors of tumor invasion. At least two patterns of $\mathrm{CD} 10^{+}$stromal cell infiltration were seen, indicating the possibility of several mechanisms and relationships with MM development. Maguer-Satta et al. ${ }^{14)}$ suggested that CD10 is supported by two major mechanisms: 1) extracellular enzymatic activity on 
surfaces of epithelial and stromal cells; and 2) intracellular signaling that interferes with other major signaling pathways. These CD10 mechanisms regulate biological functions of normal and malignant stem cells and their environments.

We found $34 \mathrm{CD} 10^{+}$samples out of $36 \mathrm{MM} \mathrm{ca-}$ ses $(94.4 \%)$ in both or either tumor and stromal cells, compared with 8 out of 50 BMN samples $(16.0 \%)$ in stromal cells only. This method was $94 \%$ sensitive and $84 \%$ specific, with an $81 \%$ PPV and a $96 \%$ NPV. The IHC sensitivity for tumoral and/or stromal expression of CD10 is higher than that for tumoral expression only, indicating that combining tumoral and stromal CD10 expression would be the best approach for its use in diagnosis of MM.

In conclusion, this study suggests a potential utility of CD10 in differentiating advanced MM from benign melanocytic lesion. IHC CD10 expression in either tumor or stromal cells could indicate higher possibilities of malignant disease. However, CD10 staining that is negative or fine and focally positive may represent benign lesions. Although clinical and pathological evaluation is essential for making final diagnoses, immunohistochemical CD10 expression could help distinguish MM and BMN.

\section{Acknowledgments}

We would particularly like to thank Dr. Tatsunami, Unit of Medical Statistics, Faculty of Education and Culture, St. Marianna University School of Medicine, for his invaluable suggestion of statistical analyses in this study.

\section{References}

1) Braun RP, Rabinovitz HS, Oliviero M, Kopf AW, Saurat JH. Dermoscopy of pigmented skin lesions. J Am Acad Dermatol 2005; 52: 109121.

2) Rigel DS, Russak J, Friedman R. The evolution of melanoma diagnosis: 25 years beyond the ABCDs. CA Cancer J Clin 2010; 60: 301-316.

3) Yaziji H, Gown AM. Immunohistochemical markers of melanocytic tumors. Int J Surg Pathol 2003; 11: 11-15.

4) Ivan D, Prieto VG. Use of immunohistochemistry in the diagnosis of melanocytic lesions: applications and pitfalls. Future Oncol 2010; 6: 1163-1175.

5) Ordonez NG. Value of melanocytic-associated immunohistochemical markers in the diagnosis of malignant melanoma: a review and update.
Hum Pathol 2014; 45: 191-205.

6) Kanitakis J, Narvaez D, Claudy A. Differential expression of the CD10 antigen (neutral endopeptidase) in primary versus metastatic malignant melanomas of the skin. Melanoma Res 2002; 12: 241-244.

7) Bilalovic N, Sandstad B, Golouh R, Nesland JM, Selak I, Torlakovic EE. CD10 protein expression in tumor and stromal cells of malignant melanoma is associated with tumor progression. Mod Pathol 2004; 17: 1251-1258.

8) Velazquez EF, Yancovitz M, Pavlick A, Berman R, Shapiro R, Bogunovic D, O’Neill D, Yu YL, Spira J, Christos PJ, Zhou XK, Mazumdar M, Nanus DM, Liebes L, Bhardwaj N, Polsky D, Osman I. Clinical relevance of neutral endopeptidase (NEP/CD10) in melanoma. J Transl Med 2007; 5: 2.

9) Thomas-Pfaab M, Annereau JP, Munsch C, Guilbaud N, Garrido I, Paul C, Brousset P, Lamant L, Meyer N. CD10 expression by melanoma cells is associated with aggressive behavior in vitro and predicts rapid metastatic progression in humans. J Dermatol Sci 2013; 69: 105-113.

10) McIntosh GG, Lodge AJ, Watson P, Hall AG, Wood K, Anderson JJ, Angus B, Horne CH, Milton ID. NCL-CD10-270: a new monoclonal antibody recognizing CD10 in paraffin-embedded tissue. Am J Pathol 1999; 154: 77-82.

11) Chu P, Arber DA. Paraffin-section detection of CD10 in 505 nonhematopoietic neoplasms. Frequent expression in renal cell carcinoma and endometrial stromal sarcoma. Am J Clin Pathol 2000; 113: 374-382.

12) Chu PG, Arber DA, Weiss LM, Chang KL. Utility of CD10 in distinguishing between endometrial stromal sarcoma and uterine smooth muscle tumors: an immunohistochemical comparison of 34 cases. Mod Pathol 2001; 14: 465-471.

13) Kanner WA, Brill LB, 2nd, Patterson JW, Wick MR. CD10, p63 and CD99 expression in the differential diagnosis of atypical fibroxanthoma, spindle cell squamous cell carcinoma and desmoplastic melanoma. J Cutan Pathol 2010; 37: 744-750.

14) Maguer-Satta V, Besancon $R$, Bachelard-Cascales E. Concise review: neutral endopeptidase (CD10): a multifaceted environment actor in stem cells, physiological mechanisms, and cancer. Stem Cells 2011; 29: 389-396.

15) Jongeneel CV, Quackenbush EJ, Ronco P, Ver- 
roust P, Carrel S, Letarte M. Common acute lymphoblastic leukemia antigen expressed on leukemia and melanoma cell lines has neutral endopeptidase activity. J Clin Invest 1989; 83: 713-717.

16) Carrel S, Schreyer M, Gross N, Zografos L. Surface antigenic profile of uveal melanoma lesions analysed with a panel of monoclonal antibodies directed against cutaneous melanoma. Anticancer Res 1990; 10: 81-89.

17) Carrel S, Zografos L, Schreyer M, Rimoldi D. Expression of CALLA/CD10 on human melanoma cells. Melanoma Res 1993; 3: 319-323.

18) Kanitakis J, Bourchany D, Claudy A. Expression of the CD10 antigen (neutral endopeptidase) by mesenchymal tumors of the skin. Anticancer Res 2000; 20: 3539-3544.

19) Bertucci F, Pages C, Finetti P, Rochaix P, Lamant L, Devilard E, Nguyen C, Houlgatte R, Birnbaum D, Xerri L, Brousset P. Gene expression profiling of human melanoma cell lines with distinct metastatic potential identifies new progression markers. Anticancer Res 2007; 27: 34413449.

20) Oba J, Nakahara T, Hayashida $S$, Kido M, Xie L, Takahara M, Uchi H, Miyazaki S, Abe T, Hagihara A, Moroi Y, Furue M. Expression of CD10 predicts tumor progression and unfavorable prognosis in malignant melanoma. J Am Acad Dermatol 2011; 65: 1152-1160.

21) Momose M, Ota H, Hayama M. Re-evaluation of melanin bleaching using warm diluted hydrogen peroxide for histopathological analysis. Pathol Int 2011; 61: 345-350.

22) Annessi G, Bono R, Sampogna F, Faraggiana T, Abeni D. Sensitivity, specificity, and diagnostic accuracy of three dermoscopic algorithmic methods in the diagnosis of doubtful melanocytic lesions: the importance of light brown structureless areas in differentiating atypical melanocytic nevi from thin melanomas. J Am Acad Dermatol 2007; 56: 759-767.

23) Moritani S, Kushima R, Sugihara H, Bamba M, Kobayashi TK, Hattori T. Availability of CD10 immunohistochemistry as a marker of breast myoepithelial cells on paraffin sections. Mod
Pathol 2002; 15: 397-405.

24) Kubiak-Wlekly A, Niemir ZI. [Neprilysinstructure of the gene and protein product and the localization of expression]. Pol Merkur Lekarski 2009; 27: 48-50.

25) McCluggage WG, Sumathi VP, Maxwell P. CD10 is a sensitive and diagnostically useful immunohistochemical marker of normal endometrial stroma and of endometrial stromal neoplasms. Histopathology 2001; 39: 273-278.

26) Attygalle A, Al-Jehani R, Diss TC, Munson P, Liu $\mathrm{H}, \mathrm{Du} \mathrm{MQ}$, Isaacson PG, Dogan A. Neoplastic $\mathrm{T}$ cells in angioimmunoblastic $\mathrm{T}$-cell lymphoma express CD10. Blood 2002; 99: 627633.

27) Buonaccorsi JN, Plaza JA. Role of CD10, widespectrum keratin, p63, and podoplanin in the distinction of epithelioid and spindle cell tumors of the skin: an immunohistochemical study of 81 cases. Am J Dermatopathol 2012; 34: 404411.

28) Iwaya K, Ogawa H, Izumi M, Kuroda M, Mukai K. Stromal expression of CD10 in invasive breast carcinoma: a new predictor of clinical outcome. Virchows Arch 2002; 440: 589-593.

29) Makretsov NA, Hayes M, Carter BA, Dabiri S, Gilks CB, Huntsman DG. Stromal CD10 expression in invasive breast carcinoma correlates with poor prognosis, estrogen receptor negativity, and high grade. Mod Pathol 2007; 20: 84-89.

30) Jana SH, Jha BM, Patel C, Jana D, Agarwal A. CD10-a new prognostic stromal marker in breast carcinoma, its utility, limitations and role in breast cancer pathogenesis. Indian J Pathol Microbiol 2014; 57: 530-536.

31) Ogawa H, Iwaya K, Izumi M, Kuroda M, Serizawa H, Koyanagi Y, Mukai K. Expression of CD10 by stromal cells during colorectal tumor development. Hum Pathol 2002; 33: 806-811.

32) Xie L, Moroi Y, Takahara M, Tsuji G, Oba J, Hayashida S, Takeuchi S, Shan B, Uchi H, Furue M. CD10 expressed by fibroblasts and melanoma cells degrades endothelin-1 secreted by human keratinocytes. Eur J Dermatol 2011; 21: 505-509. 